\title{
MEDIUM AND ATMOSPHERIC PRESSURE PLASMA TREATMENT FOR IMPROVEMENT OF ADHESION OF PDMS USED FOR FLEXIBLE AND STRETCHABLE ELECTRONICS
}

\author{
N. DE GEYTER ${ }^{1}$, R. MORENT ${ }^{1}$, F. AXISA ${ }^{2}$, E. DE LEERSNYDER ${ }^{2}$, C. LEYS ${ }^{1}$, \\ J. VANFLETEREN ${ }^{2}$, N. DE SMET ${ }^{3}$, M. RYMARCZYK-MACHAL ${ }^{3}$ AND E. SCHACHT $^{3}$
}

\begin{abstract}
${ }^{1}$ Department of Applied Physics, Research Unit Plasma Technology, Faculty of Engineering, Ghent University, Jozef Plateaustraat 22, 9000 Ghent, Belgium, e-mail:Nathalie.DeGeyter@UGent.be ${ }^{2}$ Department of Electronics and Information Systems, TFCG Microsystems, Faculty of Engineering, Ghent University, Technologiepark 914, Grote Steenweg Noord, 9052 Ghent, Belgium

${ }^{3}$ Department of Organic Chemistry, Biomaterials Research Group, Faculty of Sciences, Ghent University, Krijgslaan 281 S4, 9000 Ghent, Belgium
\end{abstract}

\section{Introduction}

It is believed that in the near future, citizens will carry along more and more electronic systems near the body or even inside the body. These electronic circuits should not lead to a decrease in comfort for the user and should therefore be compact and light-weighted [1]. Moreover, the circuits should be soft, stretchable and elastic so that they can take the shape of the object in which they are integrated to guarantee maximal comfort. In addition, when implanting electronic systems inside the human body, these systems should be highly biocompatible [2].

Stretchable electronics consist of a moulded matrix of polydimethylsiloxane (PDMS) with integrated circuits connected by stretchable interconnections. Today metals (cupper, nickel and gold) are the best option to realize this type of interconnection with high performance and low cost. However, metals are not intrinsically stretchable, therefore, a suitable design such as a meander shaped structure is necessary [2]. The general production process of the stretchable electronics is shown in Figure 1. A photoresist is spin coated on a copper foil and patterned with the desired conductor shape. In the next step, a nickel seed layer followed by a $4 \mu \mathrm{m}$ thick gold layer and a nickel-gold finish are electroplated and the photoresist is dissolved. The components are then assembled and connected to the interconnections by gluing or soldering. In a next step, the sample is overmoulded with viscous PDMS and thermally cured. The copper foil is removed, resulting in a thin film of PDMS with the interconnections and components at the surface. Finally, the electronic structures are encapsulated by overmoulding the sample with viscous silicone [1-3]. This technology has been developed in the frame of the Bioflex programme (IWT SBOBioflex (contract 04101)).

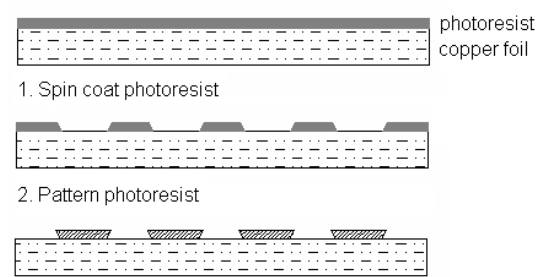

3. Electroplate gold-nickel metal

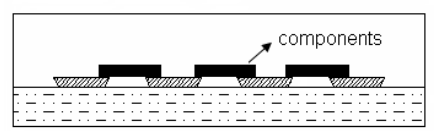

4. Assemble components and overmould PDMS

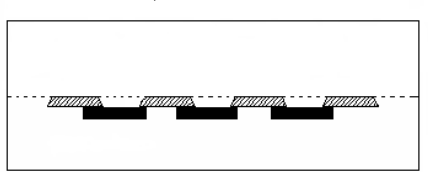

5. Remove copper foil, invert sample and place another PDMS layer on top

Figure 1. Process sequence for the fabrication of stretchable electronics

The metals inside the electronic circuit should not leak out in order to obtain a highly biocompatible system. Therefore, an excellent adhesion between the two PDMS layers is of great importance. However, PDMS has a very low surface energy, which makes the formation of strong and permanent bonds to this material impossible without some kind of surface treatment [4]. Adhesive bonding of materials is being frequently utilized; however, this technique has some important limitations, such as a long bonding time and a low resistance to heat and chemicals [5]. Recent studies [6-7] have shown that bonding of polymers to polymers can be achieved without the necessity of any adhesive by treating the involved surfaces with a non-thermal plasma. In this paper, PDMS films are plasma-treated with two types of non-thermal plasmas to enhance their adhesion: a dielectric barrier discharge (DBD) operated in air at medium pressure $(5.0 \mathrm{kPa})$ and a remote DC glow discharge operated in air at atmospheric pressure. The effects of both 
plasma treatments on a PDMS film are studied using contact angle measurements and X-ray Photoelectron Spectroscopy (XPS). This work also investigates whether the changes induced by both plasma treatments contribute to an improvement of the adhesion properties of the PDMS surfaces.

\section{Experimental details}

\subsection{Materials}

The PDMS films are manufactured from a biomedical-grade silicone rubber kit (Silastic ${ }^{\circledR}$ - MDX4-4210 Dow Corning), consisting of an elastomer component and a curing agent. The elastomer component consist of a dimethylsiloxane polymer, a reinforcing silica and a platinum catalyst, while the curing agent component consists of a dimethylsiloxane polymer, an inhibitor and a siloxane crosslinker. The elastomer component and the curing agent from the kit were thoroughly blended in a 10:1 ratio after which the air bubbles were removed by vacuum. The silicone mix was placed into a mould (to produce $250 \mu \mathrm{m}$ thick sheets) and afterwards, the silicone is cured in air by placing the mould in an oven of $150^{\circ}$ (30 minutes). PDMS consists of an inorganic backbone of alternating silicon and oxygen atoms and methyl groups are attached to the silicon atoms forming the repeating unit in the polymer (see Figure 2).

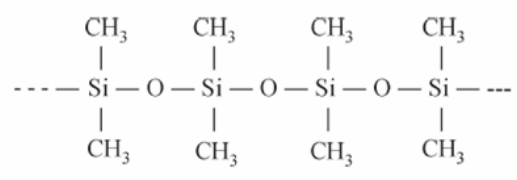

Figure 2. Chemical structure of PDMS

\subsection{DBD set-up}

A schematic diagram of the DBD configuration is depicted in Figure 3. Two circular copper electrodes (diameter $=7 \mathrm{~cm}$ ) are placed within a cylindrical enclosure. Both electrodes are covered with a glass plate (thickness $=2 \mathrm{~mm}$ ) and the gas gap between the glass plates is $3 \mathrm{~mm}$. The upper electrode is connected to an $\mathrm{AC}$ power source with a frequency of $50 \mathrm{kHz}$ and the lower electrode to earth through a resistor of $100 \Omega$. The plasma power is kept constant at 6.6 $\mathrm{W}$, leading to a power density of $0.143 \mathrm{~W} / \mathrm{cm}^{3}$. Between the electrodes, air (Air Liquide - Alphagaz 1) is fed into the system at a rate of $200 \mathrm{sccm}$. A rotary vane pump is attached to the gas outlet and the pressure in the chamber is maintained at $5.0 \mathrm{kPa}$ by the use of a valve. During the experiments, a PDMS film is placed on the lower glass plate. Plasma treatment is then carried out for varying treatment

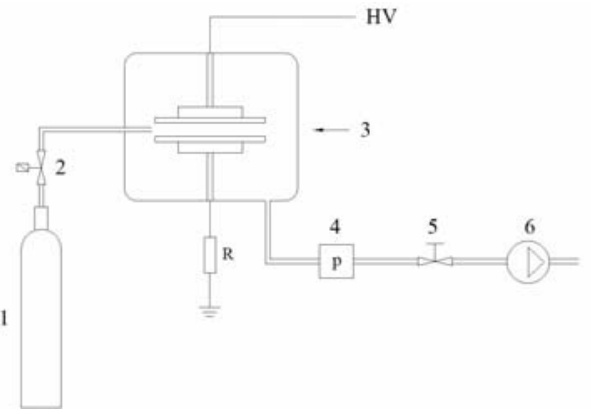

Figure 3. Experimental set-up of the DBD (1. gas cylinder, 2. mass-flow controller, 3. plasma chamber, 4. pressure gauge, 5. needle valve, 6. pump) times.

\subsection{Remote DC glow set-up}

The experimental set-up used for the remote plasma treatment at atmospheric pressure is similar to that reported in [8] and is shown in Figure 4. The atmospheric pressure plasma source consists of an aluminium plate anode and a single row of 28 cathode electrode elements oriented in parallel to the treated surface. Each of these electrode elements is a stainless steel pin, ballasted with a resistor of 1.5 M $\Omega$ (Caddock - MX440). The aluminium plate anode is connected to a DC high voltage power supply with output voltage up to $30 \mathrm{kV}$, while the cathode electrode elements are connected to earth. The electrode gap is $10 \mathrm{~mm}$ and the discharge power is kept constant at $126.7 \mathrm{~W}$, leading to a power density of approximately

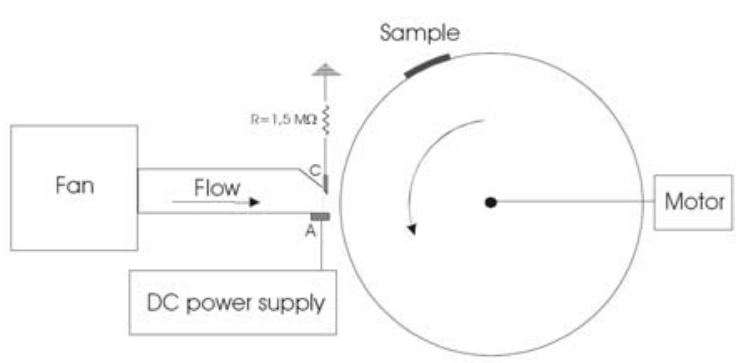

Figure 4. Experimental set-up of the DC glow discharge ( $C=$ cathode pins, $A=$ anode plate) 
$9 \mathrm{~W} / \mathrm{cm}^{3}$. The non-thermal plasma is generated between the two electrodes and a fast airflow of $40 \mathrm{~m} / \mathrm{s}$ produced by a fan (Ventomatic - CMT31M) transports the plasma-produced reactive particles towards the PDMS sample to be treated. To diminish any losses in reactive species during their transportation to the treated surface, the distance between the output of the plasma source and the PDMS sample is only $3 \mathrm{~mm}$. The sample is mounted on a rotating drum of about $0.40 \mathrm{~m}$ in diameter, in order to simulate in-line processing at variable line speeds. The results presented below are for so-called cyclical treatment: this means that the sample passes once or a number of times through the exit region of the plasma source, where it is exposed to the flowing afterglow.

\section{Results}

\subsection{Contact angle measurements}

Contact angle measurement is the ideal method to characterize the surface wettability. The contact angles are obtained with a contact-angle goniometer (EasyDrop - Krüss) using the sessile drop method. Distilled water is used as working liquid and the volume of the water drops is maintained at $2 \mu$. The values of the contact angles shown in this paper are the average of at least 8 measured values and the standard deviation on the average contact angles is smaller than $2 \%$.

In order to obtain the maximal adhesion between two PDMS layers, the wettability of the PDMS surfaces should be increased to the highest possible level. Figure 5 and 6 show the evolution of the contact angle of the PDMS film as a function of plasma treatment time after DBD and DC glow treatment respectively.

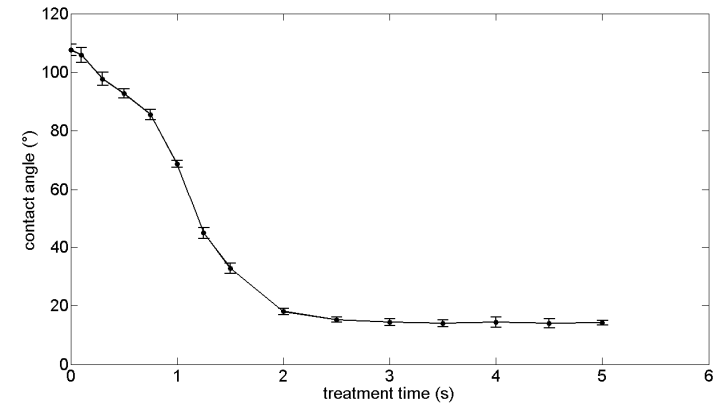

Figure 5. Evolution of the contact angle on DBD-treated PDMS film as a function of treatment time

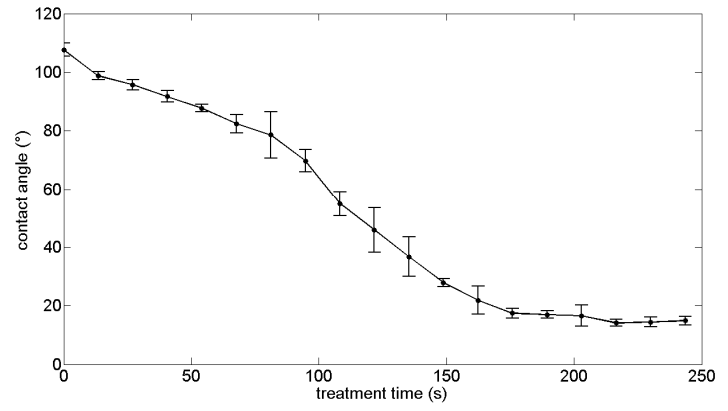

Figure 6. Evolution of the contact angle on DC glow-treated PDMS film as a function of treatment time

As shown in Figure 5 and 6, the contact angle of the PDMS film is found to change from $107.6^{\circ}$ for the untreated sample to the lowest value $14.4^{\circ}$ after 3 seconds of DBD treatment and after 216 seconds of DC glow treatment. However, when the PDMS film is treated for a longer time, the contact angle does not change anymore as a function of treatment time. This shows that there is a saturation of the plasma effect on the PDMS film. The large decrease in contact angle after both plasma treatments demonstrates the strongly increased wettability induced by the air DBD and DC glow treatment. As shown in Figure 5 and 6 , the time to reach the maximal wettability after DBD treatment is much smaller compared to the atmospheric DC glow discharge. This can be explained by the fact that the DBD treatment is an active plasma treatment, meaning that the sample is placed between the electrodes leading to a high concentration of active species near the sample surface. The DC glow treatment is a remote plasma treatment: the sample is located outside the plasma region, but passes in the gas stream that runs through the plasma zone. This gas stream is loaded with radicals and other active species, however, the concentration of these active species decreases with increasing distance to the plasma region due to collisions. Compared to the active DBD plasma treatment, less plasma species are present near the sample surface, leading to a longer treatment time necessary to obtain the maximal wettability. 


\subsection{X-ray Photoelectron Spectroscopy (XPS)}

The chemical composition of the PDMS films is investigated by X-ray photoelectron spectroscopy (XPS), which is performed in a VG Escalab $220 \mathrm{XL}$ system, using non-monochromatic $\mathrm{Mg} \mathrm{K}_{\alpha}$ - radiation $(\mathrm{hv}=1253.6 \mathrm{eV})$ operated at $15 \mathrm{kV}$ and $20 \mathrm{~mA}$. The pressure in the analysing chamber is maintained at $10^{-7} \mathrm{~Pa}$ or lower during analysis. The size of the analysed area is $8 \mathrm{~mm} \times 8 \mathrm{~mm}$. Spectra are acquired at a take-off angle of $90^{\circ}$ relatively to the sample surface. The elemental composition of the untreated and the plasma-treated PDMS films is shown in Table 1. As shown in Table 1, exposure to the non-thermal plasmas increases the oxygen content, whereas the carbon content decreases and the silicon content remains practically constant at $30-33 \%$.

Table 1. Atomic composition of an untreated PDMS film and DBD and DC glow plasma-treated PDMS films

\begin{tabular}{ccccc}
\hline & $\begin{array}{c}\text { Treatment time } \\
\text { (s) }\end{array}$ & $\begin{array}{c}\text { C } \\
\text { (at\%) }\end{array}$ & $\begin{array}{c}\text { O } \\
\text { (at\%) }\end{array}$ & $\begin{array}{c}\text { Si } \\
\text { (at\%) }\end{array}$ \\
\hline Untreated & $/$ & 41.1 & 28.1 & 30.8 \\
DBD treated & 5 & 25.0 & 44.6 & 30.4 \\
DC glow treated & 230 & 19.4 & 47.7 & 32.9 \\
\hline
\end{tabular}

To obtain further insight into the molecular changes taking place during plasma treatment, curve resolution of the Si2p peak is performed. The full width at half maximum (FWHM) of all the fitted Si2p components is kept constant at $\pm 1.6 \mathrm{eV}$. Figure 7 shows (A) the Si2p peaks of the untreated PDMS sample, (B) the PDMS film after DBD plasma treatment (treatment time $=5$ seconds) and $(\mathrm{C})$ the PDMS film after DC glow plasma treatment (treatment time $=230$ seconds).
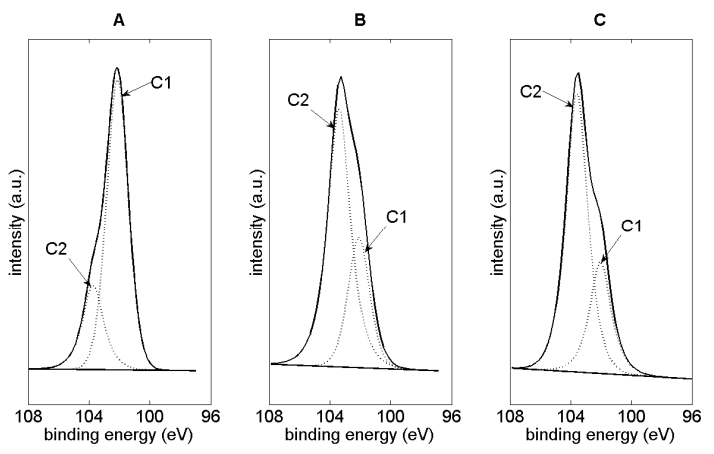

Figure 7. Si2p peaks of (A) the untreated PDMS sample, (B) the PDMS film after DBD treatment (treatment time $=5$ seconds) and (C) the PDMS film after DC glow plasma treatment (treatment time $=230$ seconds)

The Si2p peak of the untreated sample can be resolved into two peaks according to the method developed by O'Hare et al. [9]: a peak at $102.1 \mathrm{eV}(\mathrm{C} 1)$, which can be associated with silicon bound to two oxygen atoms and a peak at $103.4 \mathrm{eV}(\mathrm{C} 2)$ due to silicon bound to four oxygen atoms. This latter peak is present in the spectrum of the unexposed sample due to the reinforcing silica present in the elastomer component of the rubber kit (see section 2.1). Figure 7 shows that after DBD and DC glow treatment, the peak at $102.1 \mathrm{eV}$ decreases, while the peak at $103.4 \mathrm{eV}$ increases. The concentration of the different silicon bounds before and after plasma treatment can be seen in Table 2 . Table 2 clearly shows that during plasma treatment oxidation occurs resulting in the presence of a high concentration of silicon bound to four oxygen atoms. These results suggest the formation of linkages between $\mathrm{Si}$ and $\mathrm{O}$ atoms on the plasmatreated PDMS samples: the plasma treatment leads to the incorporation of silanol (Si-OH) groups at the expense of methyl $\left(\mathrm{CH}_{3}\right)$ groups. These silanol groups make the exposed surface highly hydrophilic since they are polar in nature [7]. 
Table 2. Concentration of the different silicon bounds on PDMS before and after plasma treatment

\begin{tabular}{|c|c|c|c|}
\hline & $\begin{array}{c}\text { Treatment time } \\
\text { (s) }\end{array}$ & $\begin{array}{c}\mathrm{CH}_{3} \\
\mathrm{l}-\mathrm{Si}-\mathrm{O} \\
\mathrm{I} \\
\mathrm{CH}_{3} \\
\mathbf{( \% )}\end{array}$ & $\begin{array}{c}0 \\
1 \\
0-s i-0 \\
1 \\
0 \\
(\%)\end{array}$ \\
\hline Untreated & / & 76.7 & 23.3 \\
\hline DBD treated & 5 & 30.6 & 69.4 \\
\hline DC glow treated & 230 & 28.5 & 71.5 \\
\hline
\end{tabular}

\subsection{Mechanical peel testing}

To test the adhesion between the PDMS layers before and after plasma treatment, a T-peel test is performed according to the international standard ISO 11339 [10]. PDMS samples are carefully cut into specimens with a width equal to $10 \mathrm{~mm}$ and a length of $60 \mathrm{~mm}$. In a next step, two PDMS films are bonded together without any adhesive over a length of $50 \mathrm{~mm}$. Five different T-peel samples are prepared: the surface treatment details of both PDMS layers can be seen in Table 3. The two unbounded ends of the flexible films are then bent in opposite directions until each end is perpendicular to the bonded assembly to form a Tshaped specimen, as shown in Figure 8. After this, the two unbounded ends are mounted into the jaws of an Instron 5543 mechanical tester. The mechanical tester is set in motion with a separation rate of 1 $\mathrm{mm} / \mathrm{min}$ and the applied force versus the distance of the grip separation

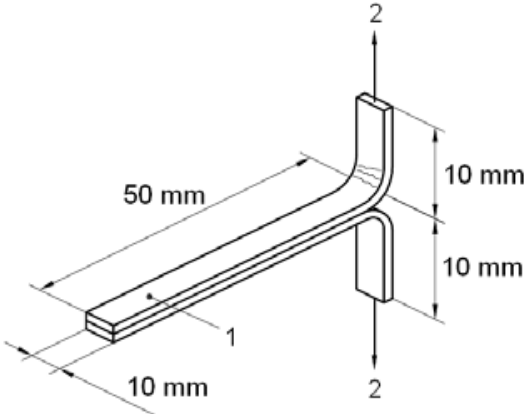

Figure 8. The T-peel test sample geometry

(1. bounded area, 2. direction of pull) [adapted from [10]] is recorded. When the applied force remains constant with increasing displacement, peeling of the two PDMS layers occurs. This constant load $\mathrm{F}(\mathrm{N})$ can be used to calculate the peel strength $(\mathrm{N} / \mathrm{m})$ using the following equation [11]:

$$
\text { peel strength }=2 \mathrm{~F} / \mathrm{W}(1)
$$

where $\mathrm{W}$ is the specimen width in meters. The calculated peel strengths are shown in Table 3 for the samples A to E, suggesting that the peel strength is not enhanced when only one PDMS layer is plasmatreated. In contrast, plasma-treating both PDMS layers results in a highly increased peel strength of up to $858 \mathrm{~N} / \mathrm{m}$ and this without the use of any adhesive. This can be explained as follows: when two PDMS layers are brought in contact, the silanol groups condense with those on another surface, resulting in the formation of Si-O-Si bonds. These covalent bonds form the basis of a tight irreversible seal between two PDMS layers [7]. When only 1 PDMS layer is plasma-treated, the formation of these Si-O-Si bonds is impossible, explaining the poor adhesion.

Table 3. Surface treatment details of test specimens used for mechanical peel testing

\begin{tabular}{lccc}
\hline & Layer 1 & Layer 2 & Peel strength (N/m) \\
\hline Sample A & untreated & untreated & 0 \\
Sample B & DBD treated $(5 \mathrm{~s})$ & untreated & 0 \\
Sample C & DC glow treated $(230 \mathrm{~s})$ & untreated & 0 \\
Sample D & DBD treated $(5 \mathrm{~s})$ & DBD treated $(5 \mathrm{~s})$ & 840 \\
Sample E & DC glow treated $(230 \mathrm{~s})$ & DC glow treated $(230 \mathrm{~s})$ & 858 \\
\hline
\end{tabular}




\section{Conclusion}

The objectives of the present research were to study the surface modifications of PDMS induced by two types of non-thermal plasmas (dielectric barrier discharge at medium pressure and DC glow discharge at atmospheric pressure) and to investigate the adhesion properties of PDMS after both plasma treatments. In the present study, several techniques have been used to investigate the consequences of both plasma treatments on PDMS films. From the obtained results, it has emerged that both plasma treatments can strongly enhance the surface wettability of PDMS: the contact angle can be decreased from $107.6^{\circ}$ for the untreated sample to the lowest value $14.4^{\circ}$. The principal chemical changes induced by both air plasma treatments are the replacement of pendant $\mathrm{CH}_{3}$-groups by silanol $(\mathrm{Si}-\mathrm{OH})$ groups. These silanol groups are polar in nature and make the surface highly hydrophilic, as observed by contact angle measurements. T-peel tests have shown that the peel strength between two untreated PDMS films is equal to $0 \mathrm{~N} / \mathrm{m}$ and this peel strength is not increased when only one PDMS layer is plasma-treated. However, when both PDMS layers are plasma-treated, the peel strength can be increased to $858 \mathrm{~N} / \mathrm{m}$ without the use of any adhesive. Taking into account the above results, one can conclude that both plasma treatments are very efficient tools to enhance the adhesion between two PDMS layers. Currently, it is investigated if this adhesion enhancement is sufficient to prevent leakage of metals into the human body.

\section{$\underline{\text { References }}$}

[1] Axisa F, Brosteaux D, De Leersnijder E, Bossuyt F, Gonzalez M, De Smet N and Vanfleteren J 2007 Proc. IEEE Polytronic 2007/6 ${ }^{\text {th }}$ international IEEE Conference on Polymers and Adhesives in Microelectronics and Photonics (Tokyo-Japan) 280-86.

[2] Brosteaux D, Axisa F, Gonzalez M and Vanfleteren J 2007 IEEE Electr. Device L. 28 552-54.

[3] Brosteaux D, Axisa F, Vanfleteren J, Carchon N and Gonzalez M 2006 Proc. MRS Spring Meeting (San Fransisco-USA).

[4] Fritz J L and Owen M J 1995 J. Adhes. 54 33-45.

[5] Katzenberg F 2005 e-Polymers nº 060.

[6] Jo B H, Van Lerberghe L M, Motsegood K M and Beebe D J 2000 J. Microelectromech. S. 9 76-81.

[7] Bhattarcharya S, Datta A, Berg J M and Gangopadhyay S 2005 J. Microelectromech. S. 14 590-97.

[8] Yu.S. Akishev, M.E. Grushin, A.P. Napartovich and N.I. Trushkin 2002 Plasmas and Polymers 7 261-89.

[9] O’Hare L-A, Parbhoo B and Leadly S R. 2004 Surf. Interface Anal. 36 1427-34.

[10] International Standard ISO 113392003 Adhesives-T-peel test for flexible-to-flexible bonded assemblies.

[11] Shenton M J, Lovell-Hoare M C and Stevens G C 2001 J. Phys. D: Appl. Phys. 34 2754-60. 